\title{
Low frequency geomagnetic field fluctuations at low latitude during the passage of a higher pressure solar wind region
}

\author{
U. Villante, P. Francia \\ Dipartimento di Fisica, Universita' dell'Aquila, L'Aquila, Italy \\ Received: 7 June 1996 / Revised: 6 January 1997 / Accepted: 20 January 1997
}

\begin{abstract}
The passage of a higher pressure solar wind region at the Earth's orbit marked the onset of low latitude $(L=1.6)$ fluctuations in the frequency range $(0.8-5.5 \mathrm{mHz})$ for both the horizontal geomagnetic field components. Spectral peaks mostly occur at the same frequencies as the spectral enhancements which appeared in the long term analysis of experimental measurements from the same station and were tentatively interpreted in terms of ground signatures of global magnetospheric modes. A comparison with simultaneous observations discussed by previous investigations allows us to conclude that the same set of frequencies is enhanced in a wide portion of the Earth's magnetosphere.
\end{abstract}

\section{Introduction}

In a recent analysis of the power spectra of the geomagnetic field components at L'Aquila (Italy, $L=1.6$, Francia and Villante, 1997) we found statistical evidence for daytime power enhancements at discrete frequencies of the order of 1.3, 1.9, 2.5, 3.6 and $4.2 \mathrm{mHz}$. These frequencies approximately correspond to those ones of events observed at auroral latitudes both in the $F$ region drift velocities (Ruohoniemi et al., 1991; Samson et al., 1992a, b; Walker et al., 1992) and in the ground geomagnetic field fluctuations (Samson et al., 1992b; Boulet et al., 1992; Ziesolleck and Mc Diarmid, 1994) and interpreted in terms of Alfvén resonances on dipole-like field lines with periods matching the eigenperiods of the driving compressional magnetospheric modes. According to models proposed by several authors (Walker et al., 1992; Samson et al., 1992a; Wright, 1994), in response to some external driving mechanism, the Earth's magnetosphere would act as a

Correspondence to: P. Francia resonant cavity or, more likely, as a wave guide along which waves, reflected by a turning point in the inner magnetosphere and by an outer boundary (such as the magnetopause), propagate antisunward. The driven field line resonances were found to be stable in frequency, within a 5-10\% margin over time intervals ranging between few hours (Walker et al., 1992) and several months (Samson et al., 1991); on the other hand, in a recent statistical survey of the occurrence of auroral latitude field line resonances, Ziesolleck and McDiarmid (1995) suggested that the apparently stable cavity/wave guide mode frequencies do not necessarily represent a unique set.

In the previous work (Francia and Villante, 1997) we found that the observed ground fluctuations might be considered permanent, solar cycle independent features which can be detected approximately at constant frequencies also at low latitudes on both the horizontal components. At low latitude similar low frequency signals have been also examined by Lilley and Bennet (1973), Ziesolleck and Chamalaun (1993) and Bloom and Singer (1995). In particular Ziesolleck and Chamalaun (1993) suggested that these waves might be interpreted in terms of global compressional modes or largescale cavity resonances trapped in the magnetosphere.

The occurrence of global magnetospheric modes can be related to several phenomena such as Kelvin-Helmholtz instability, transient dayside reconnection as well as step variations of the solar wind dynamic pressure. In this sense it is interesting to remark that the low-latitude spectral enhancements were highly amplified during intervals characterized by high solar wind velocities (Francia and Villante, 1997). On the other hand, impulsive geomagnetic disturbances such as $S I$ and $S S C$ are known to be followed by long period pulsations (5-15 min) at discrete frequencies (Nishida, 1978; Samson, 1991). Saito and Matsushita (1967) found that the frequency of the pulsations decreases with the increasing latitude; Fukunishi (1979) proposed that, between $L=3.2$ and $L=4.4$ and in the frequency range 3-100 $\mathrm{mHz}$, damped oscillations excited by $S S C$ and 
SI contain many spectral peaks and each of them appears to have a different location in latitude. Nopper et al. (1982), moreover, found that a solar wind dynamic pressure step variation triggered a worldwide impulsive disturbance that was accompanied, at $L=5$ ground stations, by field oscillations consistent with a local resonance. Similarly, Farrugia et al. (1989) found that sudden changes of the solar wind dynamic pressure were accompanied on the ground by disturbances of the geomagnetic field which were more clearly seen at auroral than at higher latitude stations; as authors remarked, higher latitude stations should be located at the feet of field lines which stretch into the polar cap and do not form a resonant cavity.

Interesting experimental results have been more recently obtained by Korotova and Sibeck (1994; K\&S hereafter) who analysed simultaneous solar wind, magnetospheric and ground observations at high, auroral and mid-latitudes observatories to investigate the magnetospheric response to a series of sharp variations of the solar wind dynamic pressure which occurred in a $3 \mathrm{~h}$ interval on November, 24, 1986. They found that the pressure variations excited coherent long period pulsations which were observed over a $30^{\circ}-50^{\circ}$ longitudinal range. As matter of fact, the dominant period of the observed fluctuations was found to depend on the observation site, although some common periods were observed in a wide range of latitudes and longitudes. As the authors remarked, none of the common periods strictly correspond to those identified by previous investigations as resonant oscillations: on the other hand, the observations were made under unusual conditions, namely with a solar wind pressure twice as great as on average conditions.

In the present note we found it interesting to analyse the geomagnetic field observations performed at L'Aquila for the same time interval as investigated by K\&S. The results of our analysis show that some peculiar signatures of the active magnetosphere observed at high, auroral and middle latitudes are still well detected at $L=1.6$. We analysed the horizontal components $(H$ and $D)$ and found several aspects of the observations which allow interesting comparisons with the results of previous investigations.

\section{Experimental results}

As in the previous study (Francia and Villante, 1997), we adopted as original data for the present investigation the $1 \mathrm{~min}$ measurements (with a resolution of $1 \mathrm{nT}$ ) of the horizontal component $H$ and of the declination $D$ (expressed in nT) obtained at the L'Aquila Geomagnetic Observatory $(L=1.6 ; \mathrm{LT}=\mathrm{UT}+1)$ run by the Isti-
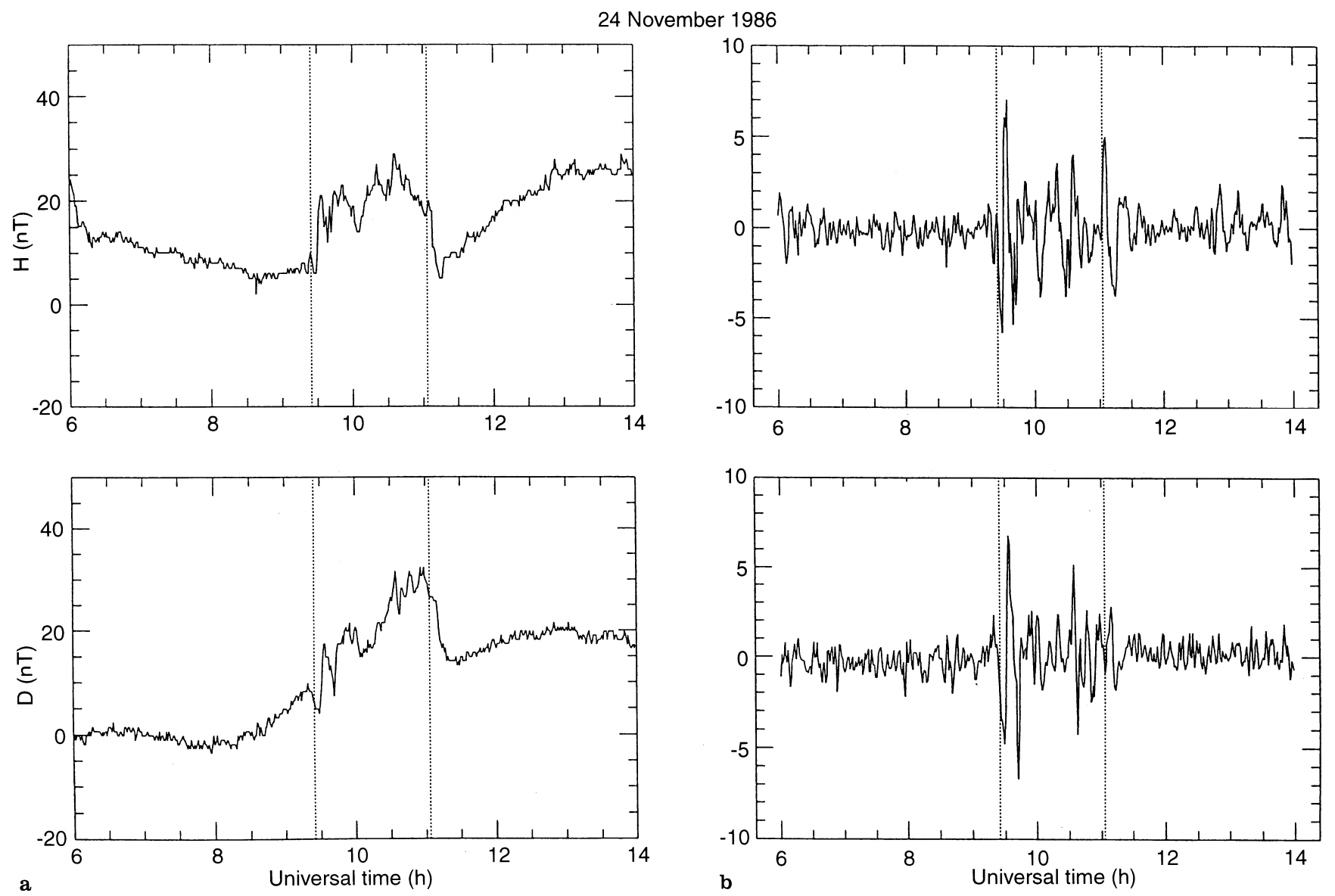

Fig. 1a,b. The $H$ and $D$ components in $\mathrm{nT}$ after subtracting the daily average value (upper and lower panel respectively) at L'Aquila Observatory on November 24, 1986. Dotted lines indicate the interval associated with the passage of the high pressure solar wind region at IMP 8 orbit 

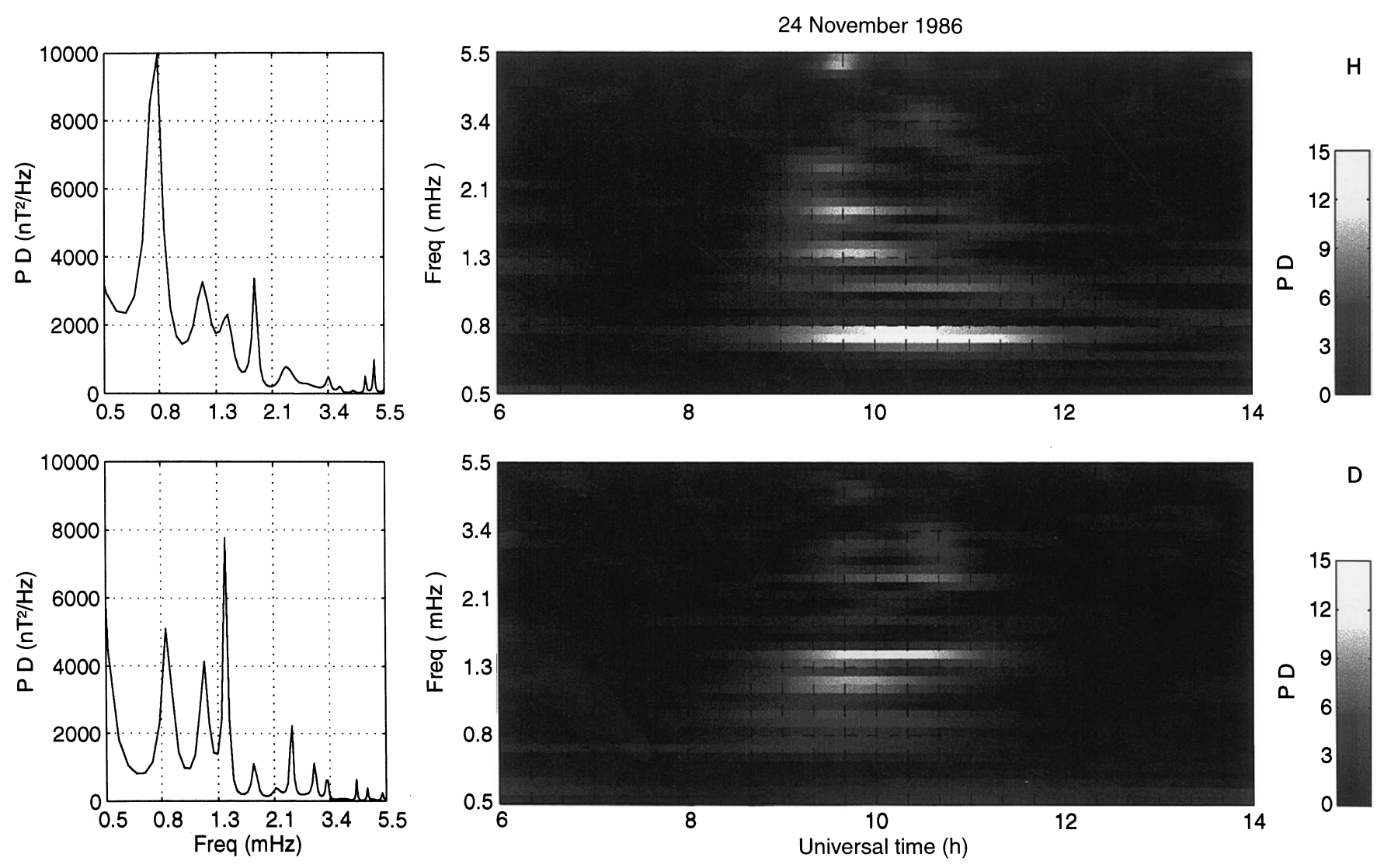

Fig. 2. The average power spectra computed by means of the maximum entropy method in the time interval 0900-1200 UT are plotted on the left for the $H$ (upper panel) and $D$ (lower panel) components. The dynamic power spectra of the two components are shown on the right for the longer time interval $0600-1400 \mathrm{UT}$; here the power is in arbitrary scale

tuto Nazionale di Geofisica. As described by Meloni et al. (1984), the instrument basically consists of a proton precession magnetometer equipped with two Helmoltz coils.

As discussed by $\mathrm{K} \& \mathrm{~S}$, in the period of interest (November, 24, 1986, 0900-1200 UT, Fig. 2 in their paper) the solar wind speed measured at the IMP 8 position upstream of the dawn bow shock varied approximately between 450 and $500 \mathrm{~km} / \mathrm{s}$. Prior to 0925 UT and after 1105 UT, the solar wind density was low $\left(5-10 \mathrm{~cm}^{-3}\right)$ and the IMF strength was high (16-20 nT). In the intervening interval, IMP 8 was imbedded in a solar wind region with higher densities $\left(20-30 \mathrm{~cm}^{-3}\right)$ and lower field strengths (8-12 nT). Step function increases of the solar wind dynamic pressure were detected approximately at 0925-0927, 0934-0937, 1005-1008, 1025-1030 UT, while a sharp pressure decrease was detected at 1101-1103 UT. The interplanetary magnetic field was generally northward except during the interval 1010-1100 UT. In the post-midnight magnetosphere a series of magnetic field compressions were detected by GOES 5 and GOES 6; the times of the magnetospheric field increases and decreases approximately correspond to those of the solar wind pressure variations. According to $\mathrm{K} \& \mathrm{~S}$, mid and high-latitude Northern Hemisphere ground observations indicated at 0925 UT an SI occurrence which marked the onset of a disturbed period at all stations; as the authors remarked, the amplitude of the dayside ground signatures decayed rapidly with decreasing latitude.

Figure 1a shows the behaviour of the geomagnetic field components at L'Aquila in the period of interest. For a better comparison with observations performed at other stations, we also show in Fig. 1b the experimental data as they appear after filtering in the same band $(0.83-5.56 \mathrm{mHz})$ proposed by K\&S. Figure 1a shows that the whole passage of the higher pressure solar wind corresponds to a sharp variation of equal duration of the low-latitude geomagnetic field elements close to the subsolar point. Dotted lines in Fig. 1a (0925-1103 UT) show that the whole ground perturbation is somewhat delayed with respect to solar wind compression region detected at the IMP 8 position. In particular both components show a prominent variation of the order of 15 nT between 0930-0934 UT, a feature which can be considered consistent with gross estimates of the propagation time $(6-7 \mathrm{~min})$ between IMP 8 position and ground (Farrugia et al., 1989). It is also interesting to remark that, when compared with observations performed at other stations (Fig. 6 in K\&S), our lowlatitude prenoon observations show a strong similarity with those obtained at higher latitude near or just before local noon (for example at Abisko, Murmansk or Sodankyla).

The power spectra evaluated for the same time interval (0900-1200 UT) investigated by K\&S are shown 
in Fig. 2 (left side) up to frequencies of $5.5 \mathrm{mHz}$. As in the previous study (Francia and Villante, 1997), the power spectra have been computed by means of the maximum entropy method at order $\mathrm{m}=30$ of error prediction filter (Vellante and Villante, 1984). The global spectra in Fig. 2 clearly show the appearance of common peaks in the $H$ and $D$ components at $0.8-0.9 \mathrm{mHz}$ (20.8-18.5 min), $\quad 1.2 \mathrm{mHz} \quad(13.9 \mathrm{~min}), \quad 1.4 \mathrm{mHz}$ (11.9 $\mathrm{min}), 1.8 \mathrm{mHz}$ (9.2 $\mathrm{min}), 2.4 \mathrm{mHz}$ (6.9 $\mathrm{min})$; some evidence for further common spectral enhancements is detected at $3.3-3.4 \mathrm{mHz}(5.1-4.9 \mathrm{~min})$ and between 4.3 and $5 \mathrm{mHz}$ (3.8 and $3.3 \mathrm{~min}$ ). A comparison with the observations performed by $\mathrm{K} \& \mathrm{~S}$ provides interesting results in that they found main spectral peaks at 1.2, 1.4 and $1.8 \mathrm{mHz}$ in a wide range of latitudes and longitudes, while peaks at approximately $2.2,2.5,3.2,4.5$ and $4.9 \mathrm{mHz}$ were also observed. We actually found major spectral peaks at the lowest frequencies $(0.8-0.9 \mathrm{mHz})$ : in this sense it is very interesting to note that, despite filtering procedure, $\mathrm{K} \& \mathrm{~S}$ (Fig. 8) also reported evidence for significant power enhancements approximately at $0.9 \mathrm{mHz}$ at several high, auroral and midlatitude stations.

The dynamic power spectra shown in the right side of Fig. 2 (as computed following the frequency-time analysis method suggested by Dziewonski et al., 1969, with $N=40$ filters centred on logarithmically equispaced frequencies and the parameter $\alpha=200$ characterizing the bandwidth of each filter) provide a rough indication of the time sequence of different spectral peaks and suggest the appearance of different spectral enhancements occurring in response to different solar wind pressure variations. For example, two different signal intensifications, mostly in the $D$ component, at the frequencies 2.4 and $3.3 \mathrm{mHz}$ are detected approximately between 0930 and 0950 UT and between 1025 and 1050 UT. In addition, within the limits of the present analysis, the results obtained for the $H$ component may allow an interesting comparison with the conclusions drawn by K\&S. They found, indeed, evidence for regular 8-10 min period oscillations soon after the SI both at auroral (0925-1020 UT) and at high (0925-1000 UT) latitudes while lower frequency modes (13-15 min) were later detected at some high-latitude stations approximately after 1000 UT. Consistently, the results of Fig. 2 (right side) show that at low latitude the highest values of the spectral peaks appear soon after the $S I$ at $1.4 \mathrm{mHz}(12 \mathrm{~min})$ and $1.8 \mathrm{mHz}(9 \mathrm{~min})$, while the peak at $1.2 \mathrm{mHz}(14 \mathrm{~min})$ emerges more clearly at a later stage.

We found also interesting to analyse in more detail the 1.4 and $1.8 \mathrm{mHz}$ fluctuations over the time interval 0925-1000 UT in which they emerge more clearly. In particular we estimated the correlation coefficient $r$ between the horizontal components (filtered in the two bands $1.1-1.6 \mathrm{mHz}$ and $1.6-2.0 \mathrm{mHz}$ ) and found that it attains maximum values (respectively $r=0.98$ and $r=0.97$, which correspond to a confidence interval greater than $99.9 \%$ ) for a 1 min time lag which can be considered indicative for an elliptical polarisation of the two modes. The polarisation patterns for the same time
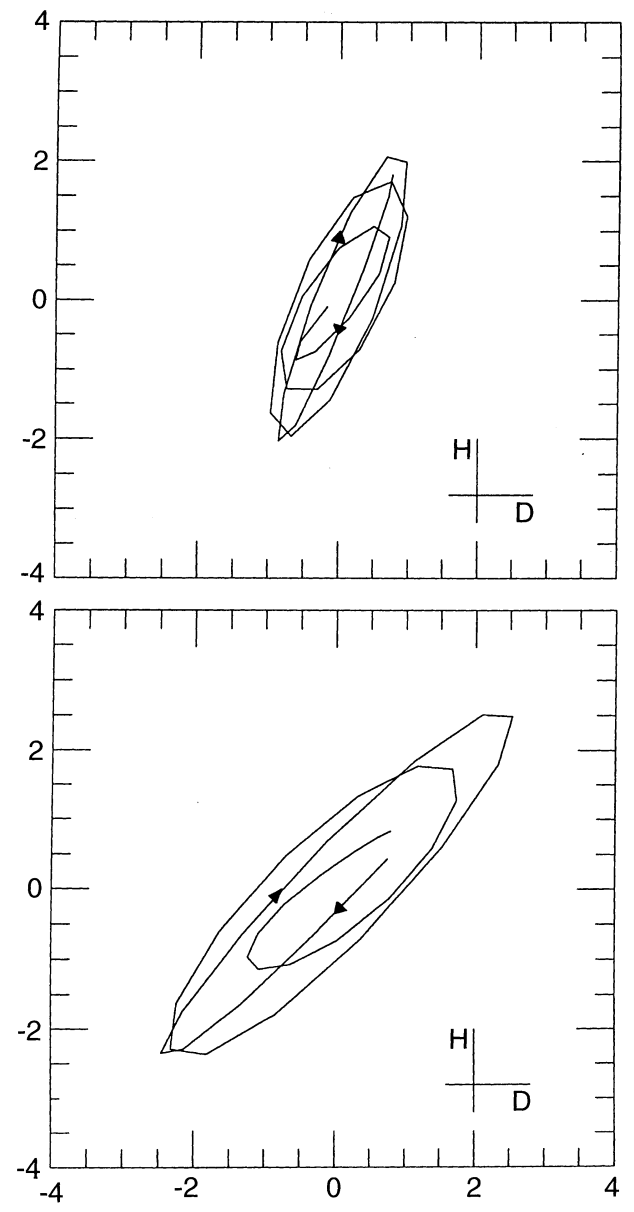

Fig. 3. The polarisation patterns for time interval 0925-1000 UT for the $1.4 \mathrm{mHz}$ (lower panel) and the $1.8 \mathrm{mhz}$ (upper panel) modes. Units are in $\mathrm{nT}$

interval (Fig. 3) show a clockwise rotation, as seen downward in the direction of the ambient magnetic field, which is the same as found by K\&S at high latitudes in the midnight and morning sectors. Nevertheless, in the further time interval (1005-1050 UT) the polarisation pattern becomes less clear and shows evidence for a counterclockwise rotation which is also consistent with the K\&S observations at high-latitude morning side stations (Mould Bay and Baker Lake).

\section{Summary and discussion}

We examined the low latitude geomagnetic field observations during an active $3 \mathrm{~h}$ interval associated with the passage of a higher pressure solar wind region at the Earth's position. When compared with suggestions of previous investigations and simultaneous higher latitude observations, the results of the present analysis allow us to draw some interesting conclusions.

a. The passage of a higher pressure solar wind region at the Earth's position is associated with a clear, equal duration variation of the low-latitude geomagnetic 
field components and a comparison among different stations shows a strong similarity with observations obtained at different latitudes near or just before local noon. In particular, the SI occurrence marked the onset of a disturbed period also at low latitudes with a considerable quantity of energy of geomagnetic field fluctuations at discrete frequencies. We found some evidence for the appearance of different spectral peaks at different times in the period of interest, suggesting that different geomagnetic field fluctuations are generated by different solar wind pressure variations.

b. When compared with higher latitude observations, the power spectra show the appearance of common peaks at several frequencies, suggesting that the coherent fluctuations observed by $\mathrm{K} \& \mathrm{~S}$ extend to a wide sector of the dayside magnetosphere. In particular, the peaks occurring at $1.2-1.4$ and $1.8 \mathrm{mHz}$, which were considered by K\&S main spectral features of a wide range of latitudes and longitudes, find clear correspondence in the present low-latitude observations. In addition, in agreement with $\mathrm{K} \& \mathrm{~S}$, who found the occurrence of different peaks between 2.2 and $5 \mathrm{mHz}$ over the whole range of latitudes, power enhancements are also detected at low latitude between 2.4 and $5 \mathrm{mHz}$. Moreover the 1.4 and 1.8 fluctuations which initiated in the time interval soon after the $S I$ event are elliptically polarized and show a clockwise rotation. The observed features seem to follow the same pattern as observed by K\&S at highlatitude stations located in the midnight and morning side sectors while, as K\&S remarked, at auroral latitudes in the morning sector the same wave train rather shows an opposite polarization.

c. A comparison with the statistical results proposed by Francia and Villante (1997) also provides interesting conclusions. Indeed, all the observed peaks appear approximately at the same frequencies (within 5\%$10 \%)$ as the spectral enhancements identified in their long-term analysis and tentatively interpreted in terms of low-latitude signatures of global magnetospheric modes. Francia and Villante (1997) also remarked that the spectral enhancements much more clearly emerge during a two month interval characterised by high solar wind velocities. Similarly, we find that in this particular period associated with a higher dynamic pressure of the solar wind, all the spectral peaks exceed approximately by a factor of 10 the average values proposed by the statistical investigation. In addition, as proposed by Francia and Villante (1997) for dayside observations, most of the power enhancements simultaneously appear (although with different energies) in the $H$ and $D$ power spectra. Similar results were obtained by Nopper et al. (1982), Ziesolleck and Chaumalaun (1993) and Bloom and Singer (1995) who also found fluctuations on both the geomagnetic field components. It is also interesting to observe that, as in the previous statistical analysis, we found evidence for a lowest frequency mode $(0.8 \mathrm{mHz}$ in the $H$ spectra, $0.9 \mathrm{mHz}$ in the $D$ spectra, $0.7 \mathrm{mHz}$ in Francia and
Villante, 1997) which in the period of interest (despite filtering procedure) is also detected by $\mathrm{K} \& \mathrm{~S}$ in higher latitude measurements.

Acknowledgements. The authors are grateful to Dr. A. Meloni, (Istituto Nazionale di Geofisica, Roma) who made available geomagnetic field observations from L'Aquila Geomagnetic Observatory. This research activity at L'Aquila is supported by MURST ( $40 \%$ and $60 \%$ contracts) and by GIFCO/CNR.

Topical Editor K.-H. Glaßmeier thanks C. W. S. Ziesolleck and $\mathrm{J}$. Verö for their help in evaluating this paper.

\section{References}

Bloom, R. M., and H. J. Singer, Diurnal trends in geomagnetic noise power in the Pc2 through Pc5 bands at low geomagnetic latitudes, J. Geophys. Res., 100, 14943, 1995.

Boulet, D., M. Bradley, and T. J. Hughes, Survey of occurrences of field line resonances with frequencies of $1.3,1.9$ and $2.6 \mathrm{mHz}$ in one year of CANOPUS magnetometer observations (abstract), EoS Trans. AGU, 73(14) Spring Meet. suppl., 251, 1992.

Dziewonski, A., S. Bloch, and M. Landisman, A technique for the analysis of transient seismic signals, Bull. Seism. Soc. Am., 59, 427, 1969.

Farrugia, C. J., M. P. Freeman, S. W. H. Cowley, D. J. Southwood, M. Lockwood and A. Etemadi, Pressure driven magnetospheric motions and attendant response on the ground, Planet. Space Sci., 37, 589, 1989.

Francia, P., and U. Villante, Some evidence of ground power enhancements at frequencies of global magnetospheric modes at low latitude, Ann. Geophysicae, 15, 17, 1997.

Fukunishi, H., Latitude dependence of power spectra of magnetic pulsations near $L=4$ excited by SSC's and SI's, J. Geophys. Res., 84, 7191, 1979.

Korotova, G. I., and D. G. Sibeck, Generation of ULF magnetic pulsations in response to sudden variations in solar wind dynamic pressure, Solar Wind Source of Magnetospheric UltraLow-Frequency Waves, Geophysical Monograph 81, AGU, 265, 1994.

Lilley, F. E. M., and D. J. Bennet, Micropulsations recorded by an array of magnetic variometers, J. Geophys. Res., 78, 1603, 1973.

Meloni, A., F. Molina, P. Palangio, Q. Taccetti, and A. De Santis, Automatic digital recording of geomagnetic elements by means of a proton precession magnetometer, Geophys. Surv., 6, 339, 1984.

Nishida, A., Geomagnetic diagnosis of the magnetosphere, Phys. Chem. Space, 9, 96, 1978.

Nopper, R. W. Jr., W. J. Hughes, C. G. MacLennan, and R. L. McPherron, Impulse excited pulsations during the July 29, 1977 event, J. Geophys. Res., 87, 5911, 1982.

Rouhoniemi, J. M., R. A. Greenwald, K. B. Baker, and J. C. Samson, HF radar observations of Pc5 field line resonances in the midnight/early morning MLT sector, Geophys. Res. Lett., 96, 15697, 1991.

Saito, T, and S. Matsushita, Geomagnetic pulsations associated with sudden commencements and sudden impulses, Planet. Space Sci., 15, 573, 1967.

Samson, J. C., Geomagnetic pulsations and plasma waves in the Earth's magnetosphere, in Geomagnetism, Ed, J. A. Jacobs vol 4, 481, 1991.

Samson, J. C., R. A. Greenwald, J. M. Ruohoniemi, T. J. Hughes, and D. D. Wallis, Magnetometer and radar observations of magnetohydrodynamic cavity modes in the Earth's magnetosphere, Can. J. Phys., 69, 929, 1991.

Samson, J. C., B. G. Harrold, J. M. Ruohoniemi, R. A. Greenwald, and A. D. M. Walker, Field line resonances associated with MHD waveguides in the magnetosphere, Geophys. Res. Lett., 19, 441, 1992a. 
Samson, J. C., D. D. Wallis, T. J. Hughes, F. Creutzberg, J. M. Ruohoniemi, and R. A. Greenwald, Substorm intensifications and field line resonances in the nightside magnetosphere, J. Geophys. Res., 97, 8495, 1992b.

Vellante, M. and U. Villante, Maximum entropy spectral analysis of artificial sinusoidal signals, J. Geophys. Res., 89, 351, 1984.

Walker A. D. M., J. M. Ruohoniemi, K. B. Baker, R. A. Greenwald, and J. C. Samson, Spatial and temporal behavior of ULF pulsations observed by the Goose Bay HF radar, J. Geophys. Res., 97, 12187, 1992.

Wright A. N., Dispersion and wave coupling in inhomogeneous MHD waveguides, J. Geophys. Res., 99, 159, 1994.
Ziesolleck, C. W. S., and F. H. Chamalaun, A two-dimensional array study of low latitude Pc5 geomagnetic pulsations, J. Geophys. Res., 98, 13703, 1993.

Ziesolleck, C. W. S., and D. R. McDiarmid, Auroral latitude Pc5 field line resonances: quantized frequencies, spatial characteristics, and diurnal variation, J. Geophys. Res., 99, 5817, 1994.

Ziesolleck, C. W. S., and D. R. McDiarmid, Statistical survey of auroral latitude Pc5 spectral and polarisation characteristics, J. Geophys. Res., 100, 19299, 1995. 